\title{
UNE REMARQUE SUR LE PROBLÈME DE CAUCHY POUR L'OPÉRATEUR DIFFÉRENTIEL DE PARTIE PRINCIPALE À COEFFICIENTS POLYNOMIAUX II
}

\author{
YÛSAKU HAMADA
}

(Received June 19, 2000)

\begin{abstract}
In [H2], we have given a remark on the domain of holomorphy of the solution of the Cauchy problem for the differential operator with polynomial coefficients. In this article, we give some complements to the results of [H2].

Résumé. Dans [H2], nous avons donné une remarque sur le domaine d'holomorphie de la solution du problème de Cauchy pour l'opérateur différentiel à coefficients polynomiaux. Dans cet article, nous donnons quelques compléments des résultats de [H2].
\end{abstract}

Introduction. Leray [L] et Gårding, Kotake et Leray [GKL] ont étudié les singularités et un prolongement analytique de la solution du problème de Cauchy dans le domaine complexe. Chazy $[\mathrm{C}]$ a étudié des équations différentielles ordinaires du troisième ordre et un système d'équations différentielles ordinaires de Darboux-Halphen. (Aussi voir [AF]). [HLT] a étudié des principes de prolongements analytiques. En appliquant ces résultats, dans cet article, nous complétons des résultats de [H2] sur le domaine d'holomorphie de la solution du problème de Cauchy pour l'opérateur différentiel à coefficients polynomiaux.

1. Notations et résultats. Soit $x=\left(x_{0}, x^{\prime}\right)\left[x^{\prime}=\left(x_{1}, x^{\prime \prime}\right), x^{\prime \prime}=\left(x_{2}, \ldots, x_{n}\right)\right]$ un point de $\boldsymbol{C}^{n+1}$. On considère un opérateur différentiel d'ordre $m$, à coefficients de fonctions entières sur $\boldsymbol{C}^{n+1}$

$$
a(x, D)=\sum_{|\alpha| \leq m} a_{\alpha}(x) D^{\alpha}, \quad D_{i}=\partial / \partial x_{i}, \quad 0 \leq i \leq n,
$$

et $g(x, D)$ est sa partie principale. Nous supposons que $g(x ; 1,0, \ldots, 0) \equiv 1$.

Soit $S$ l'hyperplan $x_{0}=0$, non caractéristique.

Étudions le problème de Cauchy

$$
a(x, D) u(x)=v(x), \quad D_{0}^{h} u\left(0, x^{\prime}\right)=w_{h}\left(x^{\prime}\right), \quad 0 \leq h \leq m-1,
$$

où $v(x), w_{h}\left(x^{\prime}\right)$ sont des fonctions entières sur $\boldsymbol{C}^{n+1}$ et $\boldsymbol{C}^{n}$ respectivement. D'après le théorème de Cauchy-Kowalewski, il existe une unique solution holomorphe au voisinage de $S$ dans $\boldsymbol{C}^{n+1}$. Dans [H1, 2], nous avons donné quelques remarques sur un prolongement analytique de cette solution locale. Dans cet article, comme dans [H2], nous étudions le cas d'opérateur différentiel à coefficients polynomiaux. Nous donnons des exemples tels que les domaines d'holomorphie des solutions sont univalents dans $\boldsymbol{C}^{n+1}$ et admettent un point extérieur dans $\boldsymbol{C}^{n+1}$.

2000 Mathematics Subject Classification. Primary 35A20; Secondary 34M99. 
Considérons les problèmes de Cauchy

$$
\left\{D_{0}+\sum_{i=1}^{3} H_{i}\left(x^{\prime}\right) D_{i}\right\} U_{1, j}(x)=0, \quad\left[x=\left(x_{0}, x^{\prime}\right), x^{\prime}=\left(x_{1}, x_{2}, x_{3}\right)\right]
$$

avec les données

$$
U_{1, j}\left(0, x^{\prime}\right)=x_{j}, \quad 1 \leq j \leq 3,
$$

où

$$
\begin{aligned}
& H_{1}\left(x^{\prime}\right)=\frac{1}{2}\left[\left(x_{2}+x_{3}\right) x_{1}-x_{2} x_{3}\right] \\
& H_{2}\left(x^{\prime}\right)=\frac{1}{2}\left[\left(x_{1}+x_{3}\right) x_{2}-x_{1} x_{3}\right] \\
& H_{3}\left(x^{\prime}\right)=\frac{1}{2}\left[\left(x_{1}+x_{2}\right) x_{3}-x_{1} x_{2}\right] .
\end{aligned}
$$

Ceci concerne le système d'équations différentielles ordinaires de Darboux-Halphen ([C], $[\mathrm{AF}])$.

Considérons les problèmes de Cauchy

$$
\left\{D_{0}+x_{2} D_{1}+x_{3} D_{2}+\left(2 x_{1} x_{3}-3 x_{2}^{2}\right) D_{3}\right\} U_{2, j}(x)=0,
$$

avec les données

$$
U_{2, j}\left(0, x^{\prime}\right)=x_{j}, \quad 1 \leq j \leq 3 .
$$

Ceci concerne l'équation différentielle ordinaire de Chazy. ([C], [AF]).

Leray [L] et Gårding, Kotake et Leray [GKL] ont étudié le problème de Cauchy, lorsque la surface initiale a des points caractéristiques. Dans [H2], nous avons étudié un cas exceptionel de [L] et [GKL]. Nous précisons ici des résultats de [H2].

Considérons les problèmes de Cauchy

$$
\left\{\sum_{i=0}^{3} A_{i}\left(x^{\prime}\right) D_{i}\right\} U_{3, j}(x)=0, \quad\left[x=\left(x_{0}, x^{\prime}\right), x^{\prime}=\left(x_{1}, x_{2}, x_{3}\right)\right]
$$

avec les données

$$
U_{3, j}\left(0, x^{\prime}\right)=x_{j}, \quad 1 \leq j \leq 3
$$

où

$$
\begin{aligned}
& A_{0}\left(x^{\prime}\right)=2 x_{1}^{2}\left(1-x_{1}\right)^{2} x_{2}, \\
& A_{1}\left(x^{\prime}\right)=A_{0}\left(x^{\prime}\right) x_{2}, \\
& A_{2}\left(x^{\prime}\right)=A_{0}\left(x^{\prime}\right) x_{3}, \\
& A_{3}\left(x^{\prime}\right)=3 x_{1}^{2}\left(1-x_{1}\right)^{2} x_{3}^{2}-\left(1-x_{1}+x_{1}^{2}\right) x_{2}^{4} .
\end{aligned}
$$

Ceci concerne l'équation différentielle ordinaire de fonction modulaire. ([C], [Hi], [AF]).

Nous avons alors 
Proposition 1.1. Les domaines d'holomorphie $\mathcal{D}_{i}, 1 \leq i, j \leq 3$, des solutions $U_{i, j}(x), 1 \leq i, j \leq 3$, des problèmes (1.2) avec (1.3), (1.5) avec (1.6) et (1.7) avec (1.8) sont des domaines univalents dans $\boldsymbol{C}^{4}$. Ils admettent un point extérieur dans $\boldsymbol{C}^{4}$.

Les problèmes (1.2) avec (1.3) et (1.5) avec (1.6) se transforment en les problèmes (1.7) avec les données transformées. Dans la Section 2, nous donnons quelques préliminaires et rappelons la fonction modulaire, son équation différentielle ordinaire et des résultats de [H2]. Dans la Section 3, nous démontrons la Proposition 1.1.

2. Quelques préliminaires, la fonction modulaire et son équation différentielle ordinaire. Considérons le problème

$$
\frac{d x_{i}}{d t}=F_{i}\left(x^{\prime}\right), \quad x_{i}(0)=y_{i}, \quad 1 \leq i \leq n, \quad\left[x^{\prime}=\left(x_{1}, \ldots, x_{n}\right)\right]
$$

où les $F_{i}\left(x^{\prime}\right), 1 \leq i \leq n$, sont des fonctions holomorphes au voisinage de l'origine de $\boldsymbol{C}^{n}$. Soient $x_{i}=f_{i}\left(t, y^{\prime}\right), 1 \leq i \leq n$, la solution holomorphe du problème (2.1) pour $t$ voisin de $0, y^{\prime}$ voisin de (0), et $y_{i}=g_{i}\left(t, x^{\prime}\right), 1 \leq i \leq n$, son inverse, donc $x_{i}=f_{i}\left(t, g\left(t, x^{\prime}\right)\right), 1 \leq$ $i \leq n$.

On a alors

Lemme 2.1. $g_{i}\left(t, x^{\prime}\right)=f_{i}\left(-t, x^{\prime}\right), 1 \leq i \leq n$, pour $t$ voisin de 0 et $x^{\prime}$ voisin de l'origine de $\boldsymbol{C}^{n}$.

Considérons l'équation différentielle

$$
\left\{D_{0}+\sum_{i=1}^{n} F_{i}\left(x^{\prime}\right) D_{i}\right\} u(x)=0 .
$$

Soient $u_{j}(x), 1 \leq j \leq l$, des solutions holomorphes de (2.2) au voisinage de $x=0$ et $\left(u_{j}(0)\right)_{1 \leq j \leq l}$ est voisin de l'origine $\operatorname{de} C^{l}$. On a

LEMme 2.2. Une fonction composée $u(x)=\mathcal{F}\left(u_{1}(x), \ldots, u_{l}(x)\right), \mathcal{F}$ étant une fonction holomorphe au voisinage de l'origine de $\boldsymbol{C}^{l}$, est une solution de (2.2).

Notons $z=L(t)=\frac{a t+b}{c t+d}$ une transformation correspondant à une matrice $L=$ $\left(\begin{array}{ll}a & b \\ c & d\end{array}\right), a, b, c, d$ étant des constantes $a d-b c=1$.

On a alors

LEMME 2.3. Par une transformation

$$
z=L(t),
$$

(C) un cercle ou une droite dans le plan de $t$ se transforme en l'axe réel du plan z. Pour $\Im(a \bar{c}) \neq 0,(C)$ est le cercle de centre $p_{L}$ et de rayon $r_{L}:\left\{t ;\left|t-p_{L}\right|=r_{L}\right\}$, où

$$
p_{L}=\frac{\bar{a} d-b \bar{c}}{a \bar{c}-\bar{a} c}, \quad r_{L}=\frac{1}{|a \bar{c}-\bar{a} c|} .
$$


L'image du point $t=p_{L}$ par (2.3) est le point $z=\overline{(a / c)}$ et le point $t=-(d / c)$ se trouve sur $(C)$. Pour $\Im(a \bar{c})=0,(C)$ est la droite: $\{t ; \Im[(a \bar{d}-\bar{b} c) t+b \bar{d}]=0\}$. Supposons $\Im(b \bar{d})>0$. Le demi-plan supérieur $\{z ; \Im z>0\}$ correspond par (2.3) respectivement à

(i) pour $\Im(a \bar{c})>0$, l'extérieur de $(C)$ contenant $t=0$,

(ii) $\operatorname{pour} \Im(a \bar{c})<0$, l'intérieur de $(C)$ contenant $t=0$,

(iii) $\quad$ pour $\Im(a \bar{c})=0$, le demi-plan $\{t ; \Im[(a \bar{d}-b \bar{c}) t+b \bar{d}]>0\}$ contenant $t=0$.

On a

LEMME 2.4. Une transformation $z=S(t)$ correspondant $\grave{a} S=\left(\begin{array}{cc}\alpha & \beta \\ \gamma & \delta\end{array}\right), \alpha, \beta, \gamma$, $\delta$ étant des constantes réelles $\alpha \delta-\beta \gamma=1$, transforme le demi-plan supérieur en lui-même. Soit $\left(\begin{array}{cc}a^{\prime} & b^{\prime} \\ c^{\prime} & d^{\prime}\end{array}\right)=S L$, alors on $a \Im\left(a^{\prime} \bar{c}^{\prime}\right)=\Im(a \bar{c}), \bar{a}^{\prime} d^{\prime}-b^{\prime} \bar{c}^{\prime}=\bar{a} d-b \bar{c}, p_{S L}=p_{L}$ et
$r_{S L}=r_{L}$.

La fonction modulaire $w=\lambda(z)$ est holomorphe $\operatorname{sur}\{z ; \Im z>0\}$ et son inverse $z=v(w)$ est holomorphe sur le revêtement universel $\mathcal{R}[C \backslash\{0,1\}]$ du domaine $C \backslash\{0,1\} . \lambda(z)$ a la frontière naturelle $\{z ; \Im z=0\}$.

Nous rappelons la ramification de fonction $v(w)$.

Lemme 2.5. Si $w$ fait un tour autour de $w=1$, dans le sens positif, le germe $v(w)$ devient $\frac{v(w)-2}{2 v(w)-3}=\left(\begin{array}{ll}1 & -2 \\ 2 & -3\end{array}\right)(v(w))$. Si $w$ fait un tour autour de $w=0$, dans le sens positif, le germe $v(w)$ devient $\left(\begin{array}{ll}1 & 0 \\ 2 & 1\end{array}\right)(v(w))$.

Considérons le problème

$$
\frac{d x_{i}}{d t}=B_{i}\left(x^{\prime}\right), \quad x_{i}(0)=y_{i}, \quad 1 \leq i \leq 3,
$$

où

$$
B_{1}\left(x^{\prime}\right)=x_{2}, \quad B_{2}\left(x^{\prime}\right)=x_{3}, \quad B_{3}\left(x^{\prime}\right)=\frac{3}{2} \frac{x_{3}^{2}}{x_{2}}-\frac{\left(1-x_{1}+x_{1}^{2}\right) x_{2}^{3}}{2 x_{1}^{2}\left(1-x_{1}\right)^{2}} .
$$

La solution du problème (2.5) s'écrit sous la forme:

$$
\begin{aligned}
x_{1}=f_{1}\left(t, y^{\prime}\right)= & \lambda\left(\frac{a\left(y^{\prime}\right) t+b\left(y^{\prime}\right)}{c\left(y^{\prime}\right) t+d\left(y^{\prime}\right)}\right), \\
x_{2}=f_{2}\left(t, y^{\prime}\right)= & \lambda^{\prime}\left(\frac{a\left(y^{\prime}\right) t+b\left(y^{\prime}\right)}{c\left(y^{\prime}\right) t+d\left(y^{\prime}\right)}\right) \frac{1}{\left(c\left(y^{\prime}\right) t+d\left(y^{\prime}\right)\right)^{2}}, \\
x_{3}=f_{3}\left(t, y^{\prime}\right)= & \lambda^{\prime \prime}\left(\frac{a\left(y^{\prime}\right) t+b\left(y^{\prime}\right)}{c\left(y^{\prime}\right) t+d\left(y^{\prime}\right)}\right) \frac{1}{\left(c\left(y^{\prime}\right) t+d\left(y^{\prime}\right)\right)^{4}} \\
& -2 \lambda^{\prime}\left(\frac{a\left(y^{\prime}\right) t+b\left(y^{\prime}\right)}{c\left(y^{\prime}\right) t+d\left(y^{\prime}\right)}\right) \frac{c\left(y^{\prime}\right)}{\left(c\left(y^{\prime}\right) t+d\left(y^{\prime}\right)\right)^{3}},
\end{aligned}
$$


où $a\left(y^{\prime}\right), b\left(y^{\prime}\right), c\left(y^{\prime}\right), d\left(y^{\prime}\right)$ sont des fonctions en $y^{\prime}=\left(y_{1}, y_{2}, y_{3}\right)$ satisfaisant $a\left(y^{\prime}\right) d\left(y^{\prime}\right)-$ $b\left(y^{\prime}\right) c\left(y^{\prime}\right)=1$.

En fait, vu les données de (2.5), on a

$$
\begin{gathered}
\lambda\left(\frac{b\left(y^{\prime}\right)}{d\left(y^{\prime}\right)}\right)=y_{1}, \quad \lambda^{\prime}\left(\frac{b\left(y^{\prime}\right)}{d\left(y^{\prime}\right)}\right) \frac{1}{d\left(y^{\prime}\right)^{2}}=y_{2}, \\
\lambda^{\prime \prime}\left(\frac{b\left(y^{\prime}\right)}{d\left(y^{\prime}\right)}\right) \frac{1}{d\left(y^{\prime}\right)^{4}}-2 \lambda^{\prime}\left(\frac{b\left(y^{\prime}\right)}{d\left(y^{\prime}\right)}\right) \frac{c\left(y^{\prime}\right)}{d\left(y^{\prime}\right)^{3}}=y_{3} .
\end{gathered}
$$

Il en résulte que

$$
\begin{aligned}
& b\left(y^{\prime}\right)=v\left(y_{1}\right) d\left(y^{\prime}\right), \quad d\left(y^{\prime}\right)=\frac{\lambda^{\prime}\left(v\left(y_{1}\right)\right)^{1 / 2}}{y_{2}^{1 / 2}}, \\
& c\left(y^{\prime}\right)=\frac{\lambda^{\prime \prime}\left(v\left(y_{1}\right)\right) y_{2}^{1 / 2}}{2 \lambda^{\prime}\left(v\left(y_{1}\right)\right)^{3 / 2}}-\frac{\lambda^{\prime}\left(v\left(y_{1}\right)\right)^{1 / 2} y_{3}}{2 y_{2}^{3 / 2}}, \\
& a\left(y^{\prime}\right)=\frac{1+b\left(y^{\prime}\right) c\left(y^{\prime}\right)}{d\left(y^{\prime}\right)} .
\end{aligned}
$$

Ces fonctions $a\left(y^{\prime}\right), b\left(y^{\prime}\right), c\left(y^{\prime}\right), d\left(y^{\prime}\right)$ sont holomorphes au voisinage d'un point $y^{\prime}=$ $\left(y_{1}, y_{2}, y_{3}\right) \in(\boldsymbol{C} \backslash\{0,1\}) \times(\boldsymbol{C} \backslash\{0\}) \times \boldsymbol{C}$ et se prolongent analytiquement $\operatorname{sur} \mathcal{R}[(\boldsymbol{C} \backslash$ $\{0,1\}) \times(\boldsymbol{C} \backslash\{0\})] \times \boldsymbol{C}$. D'après le Lemme 2.1 et (2.7), l'inverse $y_{i}=g_{i}\left(t, x^{\prime}\right), 1 \leq i \leq 3$, de $x_{i}=f_{i}\left(t, y^{\prime}\right), 1 \leq i \leq 3$, est au voisinage d'un point fixé $\left(0, x^{\prime(0)}\right), x^{\prime(0)} \in(\boldsymbol{C} \backslash\{0,1\}) \times$ $(\boldsymbol{C} \backslash\{0\}) \times \boldsymbol{C}$

$$
\begin{aligned}
y_{1}=g_{1}\left(t, x^{\prime}\right)= & \lambda\left(\frac{a\left(x^{\prime}\right) t-b\left(x^{\prime}\right)}{c\left(x^{\prime}\right) t-d\left(x^{\prime}\right)}\right) \\
y_{2}=g_{2}\left(t, x^{\prime}\right)= & \lambda^{\prime}\left(\frac{a\left(x^{\prime}\right) t-b\left(x^{\prime}\right)}{c\left(x^{\prime}\right) t-d\left(x^{\prime}\right)}\right) \frac{1}{\left(c\left(x^{\prime}\right) t-d\left(x^{\prime}\right)\right)^{2}}, \\
y_{3}=g_{3}\left(t, x^{\prime}\right)= & \lambda^{\prime \prime}\left(\frac{a\left(x^{\prime}\right) t-b\left(x^{\prime}\right)}{c\left(x^{\prime}\right) t-d\left(x^{\prime}\right)}\right) \frac{1}{\left(c\left(x^{\prime}\right) t-d\left(x^{\prime}\right)\right)^{4}} \\
& +2 c\left(y^{\prime}\right) \lambda^{\prime}\left(\frac{a\left(x^{\prime}\right) t-b\left(x^{\prime}\right)}{c\left(x^{\prime}\right) t-d\left(x^{\prime}\right)}\right) \frac{1}{\left(c\left(x^{\prime}\right) t-d\left(x^{\prime}\right)\right)^{3}} .
\end{aligned}
$$

3. La preuve de la Proposition 1.1. Vu (2.9), on a d'abord

LEMME 3.1. Les solutions $U_{3, j}(x), 1 \leq j \leq 3$, des problèmes (1.7) avec (1.8) sont holomorphes au voisinage d'un point $\left(0, x^{\prime(0)}\right)$ de $\left\{x ; x_{0}=0, x^{\prime} \in(\boldsymbol{C} \backslash\{0,1\}) \times(\boldsymbol{C} \backslash\{0\})\right.$ $\times \boldsymbol{C}\}, U_{3, j}(x)=g_{j}\left(x_{0}, x^{\prime}\right), 1 \leq j \leq 3$, et donc $D_{0} U_{3,1}(x)=-U_{3,2}(x), D_{0} U_{3,2}(x)=$ $-U_{3,3}(x)$

Les Lemmes 2.2, 2.3 et 2.5 donnent

LEMME 3.2. Au voisinage d'un point $\left(0, x^{\prime(0)}\right)$, la fonction $h(x)=\frac{a\left(x^{\prime}\right) x_{0}-b\left(x^{\prime}\right)}{c\left(x^{\prime}\right) x_{0}-d\left(x^{\prime}\right)}$ est la solution d'équation (1.7) avec la donnée $v\left(x_{1}\right)$. Elle se prolonge analytiquement $\operatorname{sur}\left\{\left(x_{0}, \tilde{x}^{\prime}\right) \in \boldsymbol{C} \times \mathcal{R}[(\boldsymbol{C} \backslash\{0,1\}) \times(\boldsymbol{C} \backslash\{0\})] \times \boldsymbol{C}, c\left(\tilde{x}^{\prime}\right) x_{0}-d\left(\tilde{x}^{\prime}\right) \neq 0\right\}$. Si $x_{1}$ fait un 
tour autour de $x_{1}=1$, le germe $h(x)$ devient $\frac{h(x)-2}{2 h(x)-3}=\left(\begin{array}{ll}1 & -2 \\ 2 & -3\end{array}\right)(h(x))$ et donc $\left(\begin{array}{ll}a\left(x^{\prime}\right) & -b\left(x^{\prime}\right) \\ c\left(x^{\prime}\right) & -d\left(x^{\prime}\right)\end{array}\right)$ devient $\left(\begin{array}{ll}1 & -2 \\ 2 & -3\end{array}\right)\left(\begin{array}{ll}a\left(x^{\prime}\right) & -b\left(x^{\prime}\right) \\ c\left(x^{\prime}\right) & -d\left(x^{\prime}\right)\end{array}\right)$. Si $x_{1}$ fait un tour autour de $x_{1}=0$, le germe $h(x)$ devient $\frac{h(x)}{2 h(x)+1}=\left(\begin{array}{ll}1 & 0 \\ 2 & 1\end{array}\right)(h(x))$ et donc $\left(\begin{array}{cc}a\left(x^{\prime}\right) & -b\left(x^{\prime}\right) \\ c\left(x^{\prime}\right) & -d\left(x^{\prime}\right)\end{array}\right)$ devient $\left(\begin{array}{ll}1 & 0 \\ 2 & 1\end{array}\right)\left(\begin{array}{ll}a\left(x^{\prime}\right) & -b\left(x^{\prime}\right) \\ c\left(x^{\prime}\right) & -d\left(x^{\prime}\right)\end{array}\right)$. Si $x_{2}$ fait un tour autour de $x_{2}=0$, le germe $h(x)$ ne change pas.

Vu les Lemmes 2.2, 3.1 et (2.9), on a

Remarque 3.1. Les fonctions $a\left(\tilde{x}^{\prime}\right) x_{0}-b\left(\tilde{x}^{\prime}\right), c\left(\tilde{x}^{\prime}\right) x_{0}-d\left(\tilde{x}^{\prime}\right), a\left(\tilde{x}^{\prime}\right)$, et $c\left(\tilde{x}^{\prime}\right)$ sont aussi des solutions de (1.7), holomorphes sur $\boldsymbol{C} \times \mathcal{R}[(\boldsymbol{C} \backslash\{0,1\}) \times(\boldsymbol{C} \backslash\{0\})]$.

Vu les Lemmes 2.4 et 3.2, on a

LEMME 3.3. Les fonctions $Q\left(x^{\prime}\right)=\Im\left[a\left(x^{\prime}\right) \overline{c\left(x^{\prime}\right)}\right], M\left(x^{\prime}\right)=\overline{a\left(x^{\prime}\right)} d\left(x^{\prime}\right)-b\left(x^{\prime}\right) \overline{c\left(x^{\prime}\right)}$ et $N\left(x^{\prime}\right)=\Im\left[a\left(x^{\prime}\right) \overline{d\left(x^{\prime}\right)}\right]$ sont analytiques en les variables réelles $\Re x^{\prime}, \Im x^{\prime}$ au voisinage de $x^{\prime(0)}$. Elles sont uniformes et analytiques en $\Re x^{\prime}$, 氵 $x^{\prime}$ sur $(\boldsymbol{C} \backslash\{0,1\}) \times(\boldsymbol{C} \backslash\{0\}) \times \boldsymbol{C}$. Les fonctions $P\left(x^{\prime}\right)=i M\left(x^{\prime}\right) / 2 Q\left(x^{\prime}\right), R\left(x^{\prime}\right)=1 / 2\left|Q\left(x^{\prime}\right)\right|$ sont uniformes et analytiques en $\Re x^{\prime}, \Im x^{\prime} \operatorname{sur}\left\{x^{\prime} \in(\boldsymbol{C} \backslash\{0,1\}) \times(\boldsymbol{C} \backslash\{0\}) \times \boldsymbol{C} ; Q\left(x^{\prime}\right) \neq 0\right\}$.

Posons

$$
\begin{aligned}
\mathcal{D}_{3}= & \left\{x=\left(x_{0}, x^{\prime}\right) \in \boldsymbol{C} \times(\boldsymbol{C} \backslash\{0,1\}) \times(\boldsymbol{C} \backslash\{0\}) \times \boldsymbol{C},\right. \\
& \left|x_{0}-P\left(x^{\prime}\right)\right|>R\left(x^{\prime}\right) \text { pour } Q\left(x^{\prime}\right)>0, \\
& \left|x_{0}-P\left(x^{\prime}\right)\right|<R\left(x^{\prime}\right) \text { pour } Q\left(x^{\prime}\right)<0, \\
& \left.\Im\left[M\left(x^{\prime}\right) x_{0}\right]-N\left(x^{\prime}\right)<0 \text { pour } Q\left(x^{\prime}\right)=0\right\} .
\end{aligned}
$$

Le domaine $\mathcal{D}_{3}$ admet un point extérieur dans $C^{4}$.

Écrivons $\tilde{\mathcal{D}}_{3}=\left\{\tilde{x}=\left(x_{0}, \tilde{x}^{\prime}\right) \in \boldsymbol{C} \times \mathcal{R}[(\boldsymbol{C} \backslash\{0,1\}) \times(\boldsymbol{C} \backslash\{0\})] \times \boldsymbol{C}, x_{0} \in \mathcal{D}_{3}\left(\pi\left(\tilde{x}^{\prime}\right)\right)\right\}$, où $\mathcal{D}_{3}\left(x^{\prime}\right)=\left\{x_{0} \in \boldsymbol{C},\left(x_{0}, x^{\prime}\right) \in \mathcal{D}_{3}\right\}$ pour $x^{\prime} \in(\boldsymbol{C} \backslash\{0,1\}) \times(\boldsymbol{C} \backslash\{0\}) \times \boldsymbol{C}$ et $\pi$ est la projection naturelle de $\mathcal{R}[(\boldsymbol{C} \backslash\{0,1\}) \times(\boldsymbol{C} \backslash\{0\})] \times \boldsymbol{C}$ sur $(\boldsymbol{C} \backslash\{0,1\}) \times(\boldsymbol{C} \backslash\{0\}) \times \boldsymbol{C} . \tilde{\mathcal{D}}_{3}$ est un revêtement de $\mathcal{D}_{3}$ et $\pi\left(x_{0}, \tilde{x}^{\prime}\right)=\left(x_{0}, \pi\left(\tilde{x}^{\prime}\right)\right)$ est la projection naturelle de $\tilde{\mathcal{D}}_{3}$ sur $\mathcal{D}_{3}$, Nous faisons

Remarque 3.2. La surface $\left\{\left(x_{0}, \tilde{x}^{\prime}\right) \in \boldsymbol{C} \times \mathcal{R}[(\boldsymbol{C} \backslash\{0,1\}) \times(\boldsymbol{C} \backslash\{0\})] \times \boldsymbol{C}, c\left(\tilde{x}^{\prime}\right) x_{0}-\right.$ $\left.d\left(\tilde{x}^{\prime}\right)=0\right\}$ se trouve sur la frontière de $\tilde{\mathcal{D}}_{3}$.

Les Lemmes 2.3, 3.1 et 3.3 donnent

LEMME 3.4. Les $U_{3, j}, 1 \leq j \leq 3$, peuvent se prolonger analytiquement sur $\tilde{\mathcal{D}}_{3}$.

D'après le théorème de Cauchy-Kowalewski, le Lemme 3.1 et (2.9), on a

Lemme 3.5. (i) Pour tout $\tilde{x}^{\prime} \in \mathcal{R}[(\boldsymbol{C} \backslash\{0,1\}) \times(\boldsymbol{C} \backslash\{0\})] \times \boldsymbol{C}$, les $U_{3, j}, 1 \leq j \leq 3$, définissent les germes uniformes et holomorphes sur $\left(\mathcal{D}_{3}\left(\pi\left(\tilde{x}^{\prime}\right)\right), \tilde{x}^{\prime}\right)$ dans $\tilde{\mathcal{D}}_{3}$. 
(ii) Les $U_{3, j}, 1 \leq j \leq 3$, peuvent se prolonger analytiquement au voisinage de $\left\{\left(0, x^{\prime}\right) ; x^{\prime} \in(\boldsymbol{C} \backslash\{0,1\}) \times(\boldsymbol{C} \backslash\{0\}) \times \boldsymbol{C}\right\}$.

Fixons un point $\left(0, x^{\prime(0)}\right) \in \boldsymbol{C} \times(\boldsymbol{C} \backslash\{0,1\}) \times(\boldsymbol{C} \backslash\{0\}) \times \boldsymbol{C}$. Pour tout $x=\left(x_{0}, x^{\prime}\right) \in \mathcal{D}_{3}$, joignons le point $\left(0, x^{\prime(0)}\right)$ au point $\left(0, x^{\prime}\right)$ par des chemins $\gamma_{1}^{(k)}, k=1,2$ dans $\{0\} \times(\boldsymbol{C} \backslash$ $\{0,1\}) \times(\boldsymbol{C} \backslash\{0\}) \times \boldsymbol{C}$ et puis le point $\left(0, x^{\prime}\right)$ au point $x$ par des chemins $\gamma_{2}^{(k)}, k=1,2$ dans $\left(\mathcal{D}_{3}\left(x^{\prime}\right), x^{\prime}\right)$. Soient $\tilde{\gamma}_{1}^{(k)}$ et $\tilde{\gamma}^{(k)}$ les relèvements sur $\tilde{\mathcal{D}}_{3}$ respectifs des chemins $\gamma_{1}^{(k)}$ et $\gamma^{(k)}=\gamma_{1}^{(k)} \circ \gamma_{2}^{(k)}$, donc $\pi\left(\tilde{\gamma}_{1}^{(k)}\right)=\gamma_{1}^{(k)}$ et $\pi\left(\tilde{\gamma}^{(k)}\right)=\gamma^{(k)}, k=1,2$.

Prolongeons analytiquement les $U_{3, j}, 1 \leq j \leq 3$, le long de $\tilde{\gamma}_{1}^{(k)}, \tilde{\gamma}^{(k)}$ et désignons par $\left.\left(U_{3, j}\right)_{\tilde{\gamma}_{1}^{(k)}}\right|_{\left(0, \tilde{x}^{\prime}(k)\right.},\left.\left(U_{3, j}\right)_{\tilde{\gamma}^{(k)}}\right|_{\tilde{x}^{(k)}}$ les germes de $U_{3, j}$ respectifs aux extrémités $\left(0, \tilde{x}^{\prime(k)}\right), \tilde{x}^{(k)}$ de $\tilde{\gamma}_{1}^{(k)}, \tilde{\gamma}^{(k)}, k=1,2$. Vu le Lemme 3.5, (ii), les germes $\left.\left(U_{3, j}\right)_{\tilde{\gamma}_{1}^{(k)}}\right|_{\left(0, \tilde{x}^{\prime(k)}\right)}, k=1,2$, sont un même germe. Donc, vu le Lemme 3.5, (i), on voit que les germes $\left.\left(U_{3, j}\right)_{\tilde{\gamma}^{(k)}}\right|_{\tilde{x}^{(k)}}, k=1,2$, sont un même germe. Notons-les donc $\left.U_{3, j}\right|_{x}, 1 \leq j \leq 3$.

Ce germe satisfait la condition de continuité suivante. Tout point $x \in \mathcal{D}_{3}$ possède un voisinage $W(x)$ de $x$ sur lequel est une fonction $\left(U_{3, j}\right)_{W(x)}$ holomorphe telle que le germe $\left.\left(U_{3, j}\right)_{W(x)}\right|_{z}$ à $z$ défini par $\left(U_{3, j}\right)_{W(x)}$ est égal à $\left.U_{3, j}\right|_{z}$ pour tout $z \in W(x)$. D'après la proposition 7.1 de [HLT], les $U_{3, j}, 1 \leq j \leq 3$ peuvent se prolonger analytiquement sur $\mathcal{D}_{3}$.

Nous notons

Lemme 3.6. Les $U_{3, j}(x), 1 \leq j \leq 3$, ne sont pas holomorphes $\grave{a}\left\{x_{0}=0, x_{1}=\right.$ $0,1\} \cup\left\{x_{0}=0, x_{2}=0\right\}$.

Preuve. Supposons la $U_{3,1}(x)$ holomorphe à l'origine $0 \mathrm{de} \boldsymbol{C}^{4}$. Le développement

$$
U_{3,1}(x)=\sum_{k=0}^{\infty} a_{k}\left(x^{\prime}\right) x_{0}^{k},
$$

$a_{k}\left(x^{\prime}\right)(k \geq 0)$ étant des fonctions holomorphes au voisinage de $x^{\prime}=0$, converge uniformément au voisinage de 0 . $\mathrm{Vu}(1.7)$ et (1.8), on a $a_{0}\left(x^{\prime}\right)=x_{1}, a_{1}\left(x^{\prime}\right)=-x_{2}, a_{2}\left(x^{\prime}\right)=$ $x_{3} / 2,12 x_{1}^{2}\left(1-x_{1}\right)^{2} x_{2} a_{3}\left(x^{\prime}\right)=(-1)\left[3 x_{1}^{2}\left(1-x_{1}\right)^{2} x_{3}^{2}-\left(1-x_{1}-x_{1}^{2}\right) x_{2}^{4}\right]$. C'est impossible. Donc la $U_{3,1}(x)$ n'est pas holomorphe à l'origine. De même on démontre les autres. Ainsi elles ne peuvent pas prolonger analytiquement au delà de la frontière de $\mathcal{D}_{3}$. Ceci implique que les domaines d'holomorphie de $U_{3, j}(x), 1 \leq j \leq 3$, sont $\mathcal{D}_{3}$. Nous démontrons ainsi la Proposition 1.1 pour les $U_{3, j}(x), 1 \leq j \leq 3$.

Nous allons étudier les $U_{1, j}(x), 1 \leq j \leq 3$.

Considérons l'application birationnelle de $\left\{x^{\prime}=\left(x_{1}, x_{2}, x_{3}\right) \in \boldsymbol{C}^{3}, x_{1} \neq x_{2}, x_{1} \neq\right.$ $\left.x_{3}, x_{2} \neq x_{3}\right\}$ sur $\left\{X^{\prime}=\left(X_{1}, X_{2}, X_{3}\right) \in \boldsymbol{C}^{3}, X_{1} \neq 0,1, X_{2} \neq 0\right\}$ :

$$
\begin{aligned}
X_{1} & =X_{1}\left(x^{\prime}\right)=\left(x_{1}-x_{3}\right) /\left(x_{1}-x_{2}\right), \\
X_{2} & =X_{2}\left(x^{\prime}\right)=\left(x_{2}-x_{3}\right)\left(x_{1}-x_{3}\right) /\left(x_{1}-x_{2}\right)=\left(x_{2}-x_{3}\right) X_{1}\left(x^{\prime}\right), \\
X_{3} & =X_{3}\left(x^{\prime}\right)=\left(x_{1}+x_{2}-x_{3}\right)\left(x_{2}-x_{3}\right)\left(x_{1}-x_{3}\right) /\left(x_{1}-x_{2}\right) \\
& =\left(x_{1}+x_{2}-x_{3}\right) X_{2}\left(x^{\prime}\right),
\end{aligned}
$$


et donc

$$
\begin{aligned}
& x_{1}=x_{1}\left(X^{\prime}\right)=\frac{X_{3}}{X_{2}}-\frac{X_{2}}{X_{1}}, \\
& x_{2}=x_{2}\left(X^{\prime}\right)=\frac{X_{3}}{X_{2}}+\frac{X_{2}}{1-X_{1}}, \\
& x_{3}=x_{3}\left(X^{\prime}\right)=\frac{X_{3}}{X_{2}}+\frac{X_{2}}{1-X_{1}}-\frac{X_{2}}{X_{1}} .
\end{aligned}
$$

Par cette transformation, le problème de Cauchy (1.2) avec (1.3) se transforme en les problèmes de Cauchy

$$
\left\{\sum_{i=0}^{3} A_{i}\left(X^{\prime}\right) D_{X_{i}}\right\} \hat{U}_{3, j}(X)=0, \quad\left[X=\left(X_{0}, X^{\prime}\right), X^{\prime}=\left(X_{1}, X_{2}, X_{3}\right)\right]
$$

avec les données

où

$$
\begin{aligned}
& \hat{U}_{3,1}\left(0, X^{\prime}\right)=\frac{X_{3}}{X_{2}}-\frac{X_{2}}{X_{1}}, \\
& \hat{U}_{3,2}\left(0, X^{\prime}\right)=\frac{X_{3}}{X_{2}}+\frac{X_{2}}{1-X_{1}}, \\
& \hat{U}_{3,3}\left(0, X^{\prime}\right)=\frac{X_{3}}{X_{2}}+\frac{X_{2}}{1-X_{1}}-\frac{X_{2}}{X_{1}},
\end{aligned}
$$

Il en résulte que

$$
U_{1, j}(x)=\hat{U}_{3, j}\left(x_{0}, X^{\prime}\left(x^{\prime}\right)\right), \quad 1 \leq j \leq 3 .
$$

$$
\begin{aligned}
& \hat{U}_{3,1}(X)=\frac{U_{3,3}(X)}{U_{3,2}(X)}-\frac{U_{3,2}(X)}{U_{3,1}(X)}, \\
& \hat{U}_{3,2}(X)=\frac{U_{3,3}(X)}{U_{3,2}(X)}+\frac{U_{3,2}(X)}{1-U_{3,1}(X)}, \\
& \hat{U}_{3,3}(X)=\frac{U_{3,3}(X)}{U_{3,2}(X)}+\frac{U_{3,2}(X)}{1-U_{3,1}(X)}-\frac{U_{3,2}(X)}{U_{3,1}(X)} .
\end{aligned}
$$

Posons $\mathcal{E}_{1}=\left\{x=\left(x_{0}, x^{\prime}\right) \in \boldsymbol{C}^{4}, x^{\prime}=\left(x_{1}, x_{2}, x_{3}\right), x_{1} \neq x_{2}, x_{2} \neq x_{3}, x_{3} \neq x_{1}, X_{0}=\right.$ $\left.x_{0},\left(X_{0}, X^{\prime}\left(x^{\prime}\right)\right) \in \mathcal{D}_{3}\right\}$ et les $U_{1, j}(x), 1 \leq j \leq 3$, sont holomorphes sur $\mathcal{E}_{1}$.

Notons $\mathcal{D}_{1}=\left(\overline{\mathcal{E}_{1}}\right)^{(\circ)}$ l'intérieur de l'adhérance $\overline{\mathcal{E}_{1}}$ de $\mathcal{E}_{1}$. On a alors $\mathcal{D}_{1} \backslash\left\{x_{k}=x_{l}, 1 \leq\right.$ $k<l \leq 3\}=\mathcal{E}_{1}$. En fait, prenons $y \in \mathcal{D}_{1}$ et $y \notin \mathcal{E}_{1}$, donc $y \in \partial \mathcal{E}_{1}$ (la frontière de $\mathcal{E}_{1}$ ). $\mathrm{Si}$ $y \notin\left\{x_{k}=x_{l}, 1 \leq k<l \leq 3\right\}$, par la définition de $\mathcal{E}_{1}$, tout voisinage de $y$ contient un point extérieur de $\mathcal{E}_{1}$. C'est contradictoire à $y \in \mathcal{D}_{1}$.

D'autre part, d'après le théorème de Cauchy-Kowalewski, les $U_{1, j}(x), 1 \leq j \leq 3$, sont holomorphes au voisinage de $S \cap\left\{x_{k}=x_{l}, 1 \leq k<l \leq 3\right\}$. D'après le théorème de Hartogs, $U_{1, j}(x), 1 \leq j \leq 3$, sont holomorphes sur $\mathcal{D}_{1}$. On voit facilement que le domaine d'holomorphie de $U_{1, j}(x), 1 \leq j \leq 3$, est $\mathcal{D}_{1}$. $\mathcal{D}_{1}$ est un domaine univalent et il admet un point extérieur dans $\boldsymbol{C}^{4}$. Ceci démontre la Proposition 1.1 pour les $U_{1, j}(x), 1 \leq j \leq 3$.

Nous remarquons les suivants. $\mathrm{Vu}(1.2),(1.3)$ et (1.4), on a d'abord 
LEMME 3.7. $U_{1,1}\left(x_{0}, x_{i}, x_{j}, x_{k}\right)=U_{1, i}(x), U_{1,2}\left(x_{0}, x_{i}, x_{j}, x_{k}\right)=U_{1, j}(x), U_{1,3}\left(x_{0}\right.$, $\left.x_{i}, x_{j}, x_{k}\right)=U_{1, k}(x), 1 \leq i, j, k \leq 3, i \neq j, j \neq k, k \neq i$.

REMARQUE 3.3. On peut calculer directement la restriction des $U_{1, j}(x), 1 \leq j \leq 3$, $\operatorname{sur}\left\{x ; x_{k}=x_{l}, k \neq l, 1 \leq k, l, \leq 3, x \in \mathcal{D}_{1}\right\}$. En fait, par exemple, posons $U_{1,1}\left(x_{0}, x_{1}, x_{2}\right.$, $\left.x_{1}\right)=V\left(x_{0}, x_{1}, x_{2}\right)$. La $V\left(x_{0}, x_{1}, x_{2}\right)$ est la solution du problème

$$
\left[D_{0}+\frac{1}{2} x_{1}^{2} D_{1}+\left(x_{1} x_{2}-\frac{1}{2} x_{1}^{2}\right)\right] V\left(x_{0}, x_{1}, x_{2}\right)=0,
$$

avec

$$
V\left(0, x_{1}, x_{2}\right)=x_{1}
$$

Il en résulte que

$$
U_{1,1}\left(x_{0}, x_{1}, x_{2}, x_{1}\right)=V\left(x_{0}, x_{1}, x_{2}\right)=\frac{2 x_{1}}{2+x_{0} x_{1}}
$$

Enfin, nous allons étudier le problème (1.5) avec (1.6).

Considérons l'application de $\boldsymbol{C}^{3}$ sur $\boldsymbol{C}^{3}$ :

$$
\begin{aligned}
& x_{1}=x_{1}\left(X^{\prime}\right)=X_{1}+X_{2}+X_{3}, \\
& x_{2}=x_{2}\left(X^{\prime}\right)=\frac{1}{2}\left(X_{1} X_{2}+X_{2} X_{3}+X_{3} X_{1}\right), \\
& x_{3}=x_{3}\left(X^{\prime}\right)=\frac{3}{2} X_{1} X_{2} X_{3} .
\end{aligned}
$$

Soient $X_{j}\left(x^{\prime}\right), 1 \leq j \leq 3$, les déterminations de la fonction algébrique définie par

$$
\tau^{3}-x_{1} \tau^{2}+2 x_{2} \tau-\frac{2}{3} x_{3}=0
$$

à un point de $\left\{x^{\prime}=\left(x_{1}, x_{2}, x_{3}\right) \in \boldsymbol{C}^{3}, \Delta\left(x^{\prime}\right) \neq 0\right\}, \Delta\left(x^{\prime}\right)$ étant le discriminant de (3.7).

Les $X_{j}\left(x^{\prime}\right), 1 \leq j \leq 3$, se prolongent analytiquement sur leur surfaces de Riemann $\mathcal{R}_{\tau}$, c'est-à-dire le revêtement du domaine $\left\{x^{\prime}=\left(x_{1}, x_{2}, x_{3}\right) \in \boldsymbol{C}^{3}, \Delta\left(x^{\prime}\right) \neq 0\right\}$. La transformation

$$
X_{j}=X_{j}\left(\tilde{x}^{\prime}\right), \quad \tilde{x}^{\prime} \in \mathcal{R}_{\tau}, \quad 1 \leq j \leq 3,
$$

transforme $\mathcal{R}_{\tau}$ en $\left\{\left(X_{1}, X_{2}, X_{3}\right) \in C^{3}, X_{1} \neq X_{2}, X_{2} \neq X_{3}, X_{3} \neq X_{1}\right\}$. Par la transformation $x_{0}=X_{0}, x_{j}=x_{j}\left(X^{\prime}\right), 1 \leq j \leq 3$, les problèmes (1.5) avec (1.6) se transforme en les problèmes

$$
\left\{D_{X_{0}}+\sum_{i=1}^{3} H_{i}\left(X^{\prime}\right) D_{X_{i}}\right\} \mathcal{U}_{1, j}(X)=0, \quad 1 \leq j \leq 3
$$

avec les données 


$$
\begin{aligned}
& \mathcal{U}_{1,1}\left(0, X^{\prime}\right)=X_{1}+X_{2}+X_{3}, \\
& \mathcal{U}_{1,2}\left(0, X^{\prime}\right)=\frac{1}{2}\left(X_{1} X_{2}+X_{2} X_{3}+X_{3} X_{1}\right), \\
& \mathcal{U}_{1,3}\left(0, X^{\prime}\right)=\frac{3}{2} X_{1} X_{2} X_{3} .
\end{aligned}
$$

où, au voisinage d'un point $\left(0, x^{\prime(0)}\right)$ de $\left\{x_{0}=0, x^{\prime} \in \boldsymbol{C}^{3}, \Delta\left(x^{\prime}\right) \neq 0\right\}$,

$$
U_{2, j}(x)=\mathcal{U}_{1, j}\left(x_{0}, X^{\prime}\left(x^{\prime}\right)\right), \quad 1 \leq j \leq 3 .
$$

On a donc, au voisinage du point $\left(0, x^{\prime(0)}\right)$,

$$
\begin{aligned}
& U_{2,1}(x)=\sum_{j=1}^{3} U_{1, j}\left(x_{0}, X^{\prime}\left(x^{\prime}\right)\right), \\
& U_{2,2}(x)=\frac{1}{2}\left\{\sum_{1 \leq j<k \leq 3} U_{1, j}\left(x_{0}, X^{\prime}\left(x^{\prime}\right)\right) U_{1, k}\left(x_{0}, X^{\prime}\left(x^{\prime}\right)\right)\right\}, \\
& U_{2,3}(x)=\frac{3}{2} U_{1,1}\left(x_{0}, X^{\prime}\left(x^{\prime}\right)\right) U_{1,2}\left(x_{0}, X^{\prime}\left(x^{\prime}\right)\right) U_{1,3}\left(x_{0}, X^{\prime}\left(x^{\prime}\right)\right) .
\end{aligned}
$$

Prenons un point arbitraire $x^{\prime}$ de $\left\{x^{\prime} ; \Delta\left(x^{\prime}\right) \neq 0\right\}$ et $\gamma$ un chemin dans $\left\{x^{\prime} ; \Delta\left(x^{\prime}\right) \neq 0\right\}$, d'origine fixé $x^{\prime(0)}$ et d'extrémité $x^{\prime}$. Prolongeons analytiquement tout $\left(X_{i}\left(x^{\prime}\right), X_{j}\left(x^{\prime}\right)\right.$, $\left.X_{k}\left(x^{\prime}\right)\right),(1 \leq i, j, k \leq 3, i \neq j, j \neq k, k \neq i)$, le long de $\gamma$ et associons le domaine $\mathcal{E}_{2}=\left\{x ; \Delta\left(x^{\prime}\right) \neq 0,\left(x_{0}, X_{i}\left(x^{\prime}\right), X_{j}\left(x^{\prime}\right), X_{k}\left(x^{\prime}\right)\right) \in \mathcal{D}_{1}, 1 \leq i, j, k \leq 3, i \neq j, j \neq k, k \neq\right.$ i\}. Puisque, si $x^{\prime}$ fait un tour autour de $\left\{x^{\prime} ; \Delta\left(x^{\prime}\right)=0\right\},\left(X_{1}\left(x^{\prime}\right), X_{2}\left(x^{\prime}\right), X_{3}\left(x^{\prime}\right)\right)$ devient une permutation de $\left(X_{1}\left(x^{\prime}\right), X_{2}\left(x^{\prime}\right), X_{3}\left(x^{\prime}\right)\right)$, le domaine $\mathcal{E}_{2}$ est bien défini. Compte tenu du Lemme 3.7, les $U_{2, j}(x), 1 \leq j \leq 3$, sont uniformes et holomorphes sur $\mathcal{E}_{2}$.

Notons $\mathcal{D}_{2}=\left(\overline{\mathcal{E}_{2}}\right)^{(\circ)}$ l'intérieur de l'adhérance $\overline{\mathcal{E}_{2}}$ de $\mathcal{E}_{2}$. Comme dans $\mathcal{D}_{1}$, on a $\mathcal{D}_{2} \backslash$ $\left\{x^{\prime} ; \Delta\left(x^{\prime}\right)=0\right\}=\mathcal{E}_{2}$. Pour chaque point $x$ de $\mathcal{D}_{2} \cap\left\{x^{\prime} ; \Delta\left(x^{\prime}\right)=0\right\}$, il existe alors un voisinage $W(x)$ dans $\mathcal{D}_{2}$ tel que les fonctions $U_{2, j}, 1 \leq j \leq 3$, sont holomorphes, uniformes et bornées dans $W(x) \backslash\left\{x^{\prime} ; \Delta\left(x^{\prime}\right)=0\right\}$, et donc en vertu d'un théorème de Riemann, elles sont holomorphes sur $\mathcal{D}_{2}$. Bien entendu, comme dans $U_{1, j}, 1 \leq j \leq 3$, on peut le démontrer aussi, en utilisant les théorèmes de Cauchy-Kowalewski et de Hartogs. On voit facilement que le domaine d'holomorphie de $U_{2, j}, 1 \leq j \leq 3$, sont $\mathcal{D}_{2}$. $\mathcal{D}_{2}$ est un domaine univalent et il admet un point extérieur dans $\boldsymbol{C}^{4}$.

\section{RÉFÉRENCES}

[AF] M. J. Ablowitz And A. S. FoKas, Complex Variables: Introduction and Applications, Cambridge Texts in Applied Mathematics, Cambridge University Press, 1997.

[C] J. ChAZY, Sur les équations différentielles du troisième ordre et d'ordre supérieur dont l'intégrale générale a ses points critiques fixes, Acta Math. 34 (1911), 317-385 .

[GKL] L. GÅrding, T. KotAKe ET J. LeRAY, Uniformisation et développement asymptotique de la solution du problème de Cauchy linéaire à données holomorphes; analogue avec la théorie des ondes asymptotiques et approchées, Bull. Soc. Math. France 92 (1964). 263-361. 
[G] R. C. GunNing, Introduction to Holomorphic Functions of Several Variables, Vol. I, Wadsworth \& Brooks/ Cole, 1991.

[HLT] Y. Hamada, J. Leray et A. Takeuchi, Prolongements analytiques de la solution du problème de Cauchy linéaire, J. Math. Pures Appl. 64 (1985), 257-319.

[H1] Y. HAMADA, Une remarque sur le domaine d'existence de la solution du problème de Cauchy pour l'opérateur différentiel à coefficients des fonctions entières, Tôhoku Math. J. 50 (1998), 133-138.

[H2] Y. HAMADA, Une remarque sur le problème de Cauchy pour l'opérateur différentiel de partie principale à coefficients polynomiaux, Tohoku Math. J. 52 (2000), 79-94.

[Hi] E. HiLLE, Ordinary Differential Equations in the Complex Domain, John Wiley, 1976.

[L] J. LERAY, Uniformisation de la solution du problème linéaire analytique de Cauchy près de la variété qui porte les données de Cauchy (Problème de Cauchy I), Bull. Soc. Math. France 85 (1957), 389-429.

[N] T. Nishino, Theory of Functions of Several Complex Variables [Tahensu Kansu Ron] (en japonais), Univ of Tokyo Press, 1996.

[P] J. PERSSON, On the local and global non-characteristic Cauchy problem when the solutions are holomorphic functions or analytic functionals in the space variables, Ark. Mat. 9 (1971), 171-180.

[PW] P. Pongérard ET C. Wagschal, Problème de Cauchy dans des espaces de fonctions entières, J. Math. Pures Appl. 75 (1996), 409-418.

61-36 TATEKURA-CHO

SHIMOGAMO, SAK YO-KU

К YОTО, 606-0806

JAPAN 